\begin{tabular}{|c|c|c|}
\hline Beitr. Ent. & Keltern & ISSN 0005-805X \\
\hline $\mathbf{6 1}(2011) 2$ & S. $239-241$ & 10.11 .2011 \\
\hline
\end{tabular}

\title{
Dem Nestor der ungarischen klassischen Entomologie, Prof. em. Dr. László Móczár, zum Geburtstag
}

Mit 1 Figur

Jenö Papp, Klára Balázs und Holger H. Dathe

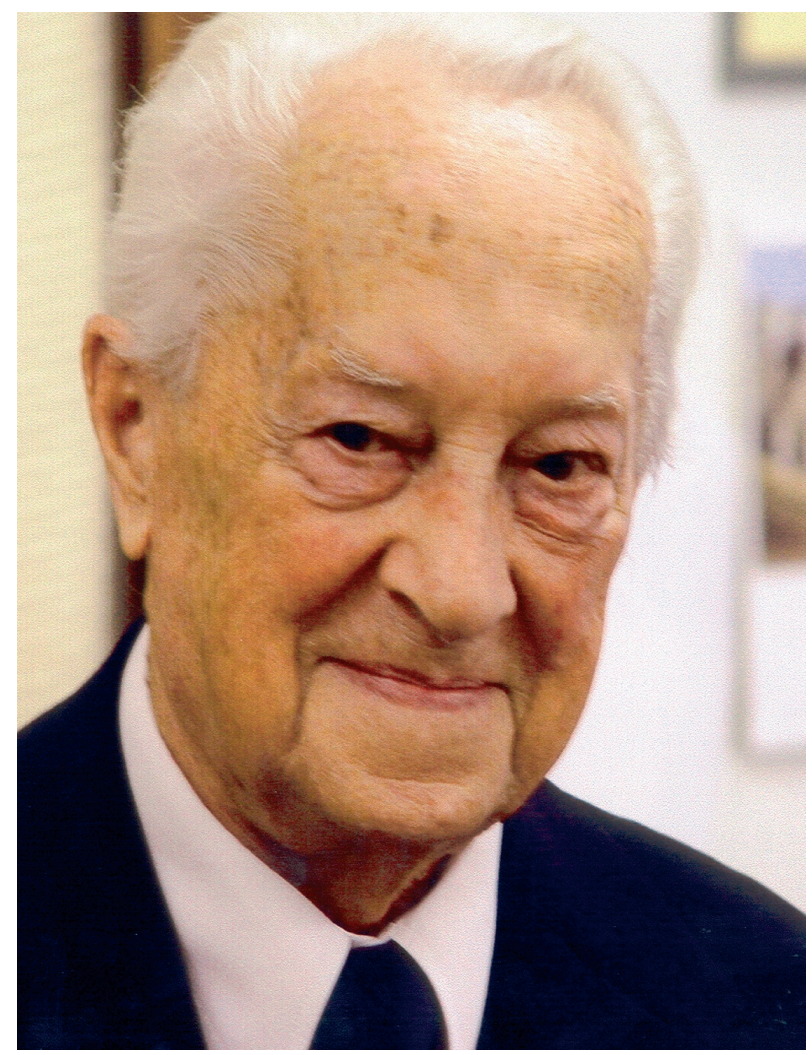

Dr. MóczÁr LÁszló, wie es in der ungarischen Originalschreibweise lautet, wurde am 10. Dezember 1914 in Kiskunfélegyhaza in der Bugac-Puszta, geboren (eingeweihten Hymenopterologen ist dieses Gebiet Inbegriff eines entomologischen Paradieses). Nach dem Studium der Zoologie in Budapest promovierte er 1937 an der Péter-Pázmány-Universität in Budapest, wo er sich 1948 auch zum Privatdozenten habilitierte. Seine berufliche Laufbahn begann er 1937 in der Zoologischen Abteilung des Naturwissenschaftlichen Landesmuseums in Budapest. Ab 1941 lehrte er an der Lehrerbildungsanstalt in Klausenburg (Cluj/Transsylvanien). Gleichzeitig war er Adjunkt an der Franz-Josef-Universität daselbst. Nach Kriegsende arbeitete er wieder 
im Ungarischen Naturwissenschaftlichen Museum in Budapest, bis er 1969 zum Professor der Zoologie an die Universität in Szeged berufen wurde. Hier beendete er 1982 seine aktive, höchst erfolgreiche Tätigkeit als Forscher und Hochschullehrer.

Schon der Vater, MikLós Móczár, war ein international ausgewiesener Hymenopterologe. Ihm folgend, wählte der Sohn die Hymenopteren zu seinem Thema, aufdem er sich in sieben Jahrzehnten zum weltweit anerkannten Aculeaten-Spezialisten emporarbeitete. Unter taxonomisch-systematischen und faunistisch-tiergeographischen Aspekten bearbeitete er die Insektenvorkommen des Karpatenbeckens, insbesondere die Goldwespen, Faltenwespen, Wegwespen und Grabwespen (Chrysidoidea, Vespoidea und Apoidea part.), zum Teil auch Blatt- und Schlupfwespen (Tenthredinoidea und Ichneumonoidea). Die zahlreichen Aufsätze über diesen Themenkreis erschienen vorwiegend in ungarischen Zeitschriften. Allein in den Folia entomologica hungarica publizierte er 27 Mitteilungen, in denen die Hymenopteren-Arten in einem damals neuartigen geographischen Rasternetz des Karpatenbeckens registriert werden. Die erwähnten Taxa bearbeitete er auch für die Serie Fauna Hungariae in mehreren Bänden. In den fünfziger Jahren führte er den Catalogus Hymenopterorum als neue Schriftenreihe ein. Ein weiterer wesentlicher Beitrag zur Bestandserfassung der ungarischen Fauna war seine umfassende Bibliographie der hymenopterologischen Literatur für Ungarn von 1897 bis 1953, unter Berücksichtigung bibliographischer Anforderungen. Auch im vorgeschrittenen Alter - im Grunde bis heute - beteiligte er sich aktiv an der Erforschung der ungarischen Nationalparks. In alle Bände zur Fauna der Nationalparks sind umfangreichen Aufsätze von ihm eingegangen. Eine Quintessenz seiner zoogeographischen Studien ist die farbige tiergeographische Karte des Karpatenbeckens, die im Ungarischen Nationalatlas (1967) erschien.

Auch Generalisten wie LÁszló MóczÁr haben ihre Spezialgruppe, in der sie „in die Tiefe gehen“. Bei MóczÁr waren es sogar mehrere; er wurde insbesondere als Weltspezialist für die Mesitiinae und Cleptinae (Familie Bethylidae/Chrysidoidea), die Ceropalinae (Familie Pompilidae) bekannt. Er revidierte die erwähnten Arten weltweit, wobei er hierzu die Sammlungen aller bedeutenden Museen studierte. Gemeinsam mit Gordon GordH publizierte er 1990 den Weltkatalog der Familie Bethylidae. Mehr als 298 neu für die Wissenschaft beschriebene Arten und mehrere neue Gattungen, die in über 242 wissenschaftlichen Arbeiten und in 10 Büchern im In- und Ausland erschienen sind, bezeugen seine unermüdliche, erfolgreiche wissenschaftliche Tätigkeit.

Auch außerhalb der Taxonomie erwarb Móczár besondere Verdienste, vor allem in der Ökologie und Ethologie. Ein herausragendes Ergebnis seiner zoozönologischen Forschungen ist die Darstellung der Abundanz- und Dominanz-Verhältnisse von Luzerneblüten besuchenden Wildbienen (Apoidea). Auf diesem Gebiet waren seine Arbeiten in Ungarn bahnbrechend. 1961 erwarb er für seine diesbezüglichen Untersuchungen den akademischen Grad Doktor der biologischen Wissenschaften der Wissenschafts-Akademie. Auf ethologischem Gebiet, speziell der Brutfürsorge, erlangten seine Beobachtungen über Handlungsreihen bei nestbauenden Eumeniden-Arten (Paragymnomerus spiricornis) grösste Bedeutung; seine Erkenntnisse wurden auch international beachtet und gingen in Lehrbücher ein. Ein Schriftenverzeichnis ist den Würdigungen von HeINRICH Wolf $(2009,2010)$ angefügt.

Ein bedeutender Abschnitt seiner Laufbahn sind die Jahre als Universitätsprofessor. Als Inhaber des Lehrstuhls für Systematische Zoologie in Szeged führte er ein neuartiges audiovisuelles Unterrichtssystem ein, wobei ihm seine jahrzehntelangen Erfahrungen als Tierfotograf, besonders als Insektenfotograf, zugutekamen. An seinem Lehrstuhl wurden gleicherweise systematische und ökologische Forschungen betrieben, zahlreiche Schüler von ihm sind heute anerkannte Zoologen, speziell Entomologen, geworden, sechs von ihnen Professoren. Aus der von ihm gegründeten Arbeitsgruppe für Ökologie ist mittlerweile der Lehrstuhl für Ökologie an der Universität Szeged entstanden. 
LÁszló MóczÁR ist auch für populärwissenschaftliche Leistungen hoch geschätzt. Unter seiner Redaktion erschien 1950 das erste ungarische Tier-Bestimmungsbuch (Állathatározó I-II.), das erweitert und mit vielen Farbfotos ergänzt 1969 und 1984 zwei weitere Auflagen erlebte. Das Buch vermittelt eine gute Übersicht über fast 6000 der in Ungarn lebenden Tierarten von den Einzellern bis zu den Säugetieren. Eine gekürzte Ausgabe dieses Buches (Kis Állathatározó, 1974) wird ebenso von Studenten der Universitäten wie auch in den Mittelschulen und von den Amateuren benutzt. Zu seinen Büchern, die sich vorwiegend mit der Entomologie befassen, gehören: Rovarok közelrÿl (Insekten aus der Nähe, 1957), Képes Allatvilág (Tierwelt in Bildern I-II, 1963), Rovarbölcsÿk (Insektenwiegen, 1987), Rovarkalauz (Insektenfÿhrer, 1990) und Rovarvilág (Insektenwelt) CD-ROM, 2003). Alle diese Bücher sind mit seinen Fotografien reich ausgestattet, wobei der Text der Bände auch von Laien gut verstanden und gern genutzt wird. Man darf ihn ohne Zweifel zu den Pionieren der Makrofotografier rechnen. Seine überaus bemerkenswerten Insekten-Aufnahmen wurden unter anderem 1952 in Salt Lake City, USA, mit einem Sonderpreis ausgezeichnet. Seine Filme haben das Interesse für die Entomologie in breiten Kreisen geweckt und als Thema weithin populär gemacht. Sein Film "Insektenwiegen" wurde 1960 beim Internationalen Zoologenkongress in Wien vorgeführt und erhielt im gleichen Jahr auch auf dem Filmfestival von Cannes eine Auszeichnung.

Die Leistungen von LÁszló MóczÁr, vor allem auf entomologischem Gebiet, wurden in Ungarn wie im Ausland vielfach anerkannt. Zahlreiche Autoren benannten 20 nova species und eine Gattung nach ihm. Über Jahrzehnte war er Vizepräsident der Ungarischen Entomologische Gesellschaft, die ihn mit der Frivaldszky-Medaille in Gold ehrte. Von der Ungarischen Biologischen Gesellschaft erhielt er den Entz- und den Gorka-Preis. Er ist Mitglied der Öster reichischen Entomologischen Gesellschaft (1975) und der Royal Entomological Society in London (1989). Außerdem ist er langjährig gewähltes Ordentliches Mitglied der Akademie der Wissenschaften St. Stephan Budapest. Vom Symposium Internationale Entomofaunisticum Europae Centralis (SIEEC, Basel 1999) wurde er mit der Ehrenmedaille ausgezeichnet. Aus Anlass seines 90. Geburtstages im Jahr 2004 verlieh ihm die Universität Szeged die Kuno-Klebelsberg-Professur, und vom Ungarischen Nationalmuseum erhielt er die Medaille Pro Studio et Fidei.

Unsere Würdigung, die hier natürlich vor allem seinem wissenschaftlichen Wirken gelten soll, wäre unzureichend, würde man seine menschlichen Werte nicht wenigstens am Rande erwähnen. Seine Liebenswürdigkeit, Hilfsbereitschaft und menschliche Ausstrahlung haben stets die Zusammenarbeit mit ihm bestimmt, die viele Kollegen dankbar erfahren durften. Die besten Wünsche aus aller Welt begleiten ihn deshalb weiter auf seinem Lebensweg.

\section{Literatur}

Wolf, H.: Professor em. Dr. László Móczár zum 95. Geburtstag. - bembiX 28 (2009): 2-13. - Entomologica Austriaca 17 (2010): 215-226 [mit Schriftenverzeichnis].

\section{Anschriften der Verfasser:}

Dr. Jenö Papp und Dr. Klára Balázs

Hungarian Natural History Museum

Baross u. 13

H-1088 Budapest, VIII, Ungarn
Prof. Dr. Holger H. Dathe

Senckenberg Deutsches Entomologisches Institut

Eberswalder Str. 90

15374 Müncheberg, Deutschland 